\title{
Communications
}

\section{Examen en vue de l'obtention de la formation approfondie en néonato- logie à adjoindre au titre de spécialiste en pédiatrie}

Lieu: Clinique de néonatologie, Centre hospitalier universitaire vaudois (CHUV), Lausanne

Date:

Examen écrit: 4.9.2018

Examen oral: 13-15.11.2018

\section{Délai d'inscription: 31.7.2018}

Vous trouverez de plus amples informations sur le site web de l'ISFM www.siwf.ch $\rightarrow$ Domaines spécialisés $\rightarrow$ Titres de spécialiste et formations approfondies (formation postgraduée) $\rightarrow$ Pédiatrie

\section{Examen de spécialiste}

Examen de spécialiste en vue de l'obtention du titre de spécialiste en prévention et santé publique

Lieu: Secrétariat SSSP, c/o polsan, Effingerstrasse 2, Berne

Date: jeudi, 13 décembre 2018

\section{Délai d'inscription: 25 octobre 2018}

Vous trouverez de plus amples informations sur le site web de l'ISFM www.siwf.ch $\rightarrow$ Domaines spécialisés $\rightarrow$ Titres de spécialiste et formations approfondies (formation postgraduée) $\rightarrow$ Prévention et santé publique

Société suisse de médecine manuelle SAMM

\section{Premier examen écrit partiel}

Selon le programme de capacité en «Médecine manuelle SAMM» du $1^{\text {er }}$ janvier 2013, les candidates et candidats à l'attestation de formation complémentaire "Médecine manuelle SAMM» doivent réussir un premier examen écrit partiel au cours du module 4.

Il faut avoir obtenu les attestations de capacité des modules 1 à 3 pour pouvoir se présenter à l'examen. La réussite de l'examen est une condition indispensable pour obtenir plus tard l'attestation de formation complémentaire "Médecine manuelle SAMM». Il est néanmoins possible de repasser l'examen deux fois.

Date: mercredi 5 septembre 2018

Heure: $9 \mathrm{~h} 30$

Lieu: Centre Loewenberg, Loewenberg 43, 3280 Morat

Vous trouvez plus d'informations sur le site web de la Société suisse de médecine manuelle SAMM www.samm.ch $\rightarrow$ Formation $\rightarrow$ Inscriptions $\rightarrow$ Premier examen écrit partiel

\section{Examen final pratique}

Selon le programme de capacité en «Médecine manuelle SAMM» du $1^{\text {er }}$ janvier 2013, les candidates et candidats à l'attestation de formation complémentaire «Médecine manuelle SAMM» doivent passer un examen final pratique après le module 8 . Pour se présenter à l'examen, les candidats doivent avoir acquis les attestations de capacité des modules 1-3 et des modules $4-8$ et réussi le premier examen partiel écrit. La réussite de l'examen final pratique est la condition indispensable pour acquérir l'attestation de formation complémentaire «Médecine manuelle SAMM». Il est possible de repasser l'examen deux fois.

Date: vendredi 26 octobre à samedi 27 octobre 2018

Heure: env. $2 \frac{1}{2}$ heures vendredi ou samedi

Lieu: Berner Fachhochschule BFH, Fachbereich Gesundheit, Stadtbachstrasse 64, 3012 Berne

Vous trouvez plus d'informations sur le site web de la Société suisse de médecine manuelle SAMM www.samm.ch $\rightarrow$ Formation $\rightarrow$ Inscriptions $\rightarrow$ Examen final pratique

Schweizerische Gesellschaft für Herz- und thorakale Gefässchirurgie (SGHC)

\section{Medtronic Young Surgeons Award}

Die Schweizerische Gesellschaft für Herz- und thorakale Gefässchirurgie verleiht eine von Medtronic gesponserte, mit $20000 \mathrm{CHF}$ dotierte Auszeichnung für eine hervorragende wissenschaftliche Arbeit aus dem Gebiet der Herz- und thorakalen Gefässchirurgie.

Die vorgelegte Arbeit darf nicht für einen anderen Preis eingereicht worden sein. Sie kann als Manuskript oder als Sonderdruck vorgelegt werden. Im Falle einer bereits erfolgten Veröffentlichung darf diese nicht länger als sechs Monate zurückliegen.

Bewerber dürfen noch keinen Facharzttitel für Herz- und thorakale Gefässchirurgie haben und müssen in der Schweiz angestellt sein.

Der Preis muss für die Ausbildung verwendet werden und wird erst nach Vorlegen des Verwendungszweckes ausbezahlt.

Bewerber reichen ihre Arbeit in vierfacher Ausführung bis 1. April 2018 dem Sekretär der Schweizerischen Gesellschaft für Herz- und thorakale Gefässchirurgie ein:

Prof. Dr. med. Christoph Huber, Chef du Service de Chirurgie Cardio-Vasculaire, HUG, Rue Gabrielle-Perret-Gentil 4, 1205 Genève

Prof. M. Genoni, Präsident SGHC Prof. Dr. med. Chr. Huber, Sekretär SGHC

Gesellschaft für die Geschichte der Schweizer Psychiatrie und Psychotherapie (GGSP)

Preis für Schweizer Psychiatriegeschichte

Die Gesellschaft für die Geschichte der Schweizer Psychiatrie und Psychotherapie (GGSP) lobt zweijährlich einen Preis für Arbeiten zur Schweizer Psychiatriegeschichte aus.

\section{Eingereichte Arbeiten}

Eingereicht werden können wissenschaftliche Originalarbeiten in der Form von Monographien, Beiträgen zu Sammelwerken oder Zeitschriftenartikel, die in den letzten zwei Jahren veröffentlicht wurden oder deren Publikation bereits schriftlich bestätigt wurde. Monographien muss eine zwei- bis fünfseitige Zusammenfassung beigelegt werden. Die Arbeiten können in deutscher, französischer oder englischer Sprache eingereicht werden.

\section{Angesprochene Autorinnen und Autoren}

Wir möchten besonders Nachwuchswissenschaftler/-innen im Bereich der Psychiatriegeschichte dazu ermutigen, ihre Arbeiten einzureichen.

Preissumme

Die Preissumme beträgt

1000 CHF für eine Arbeit von einer einzelnen Autorin/einem einzelnen Autor, 
1500 CHF für Arbeiten mit mehreren Autorinnen/Autoren.

\section{Vorgehensweise}

Die Arbeit muss elektronisch bis spätestens am 30. April 2018 an folgende E-Mail eingereicht werden: paul.hoff[at]puk.zh.ch

Die Beurteilung findet durch ein interdisziplinäres Komitee statt, worauf sämtliche Teilnehmenden über den Entscheid informiert werden.

Die Preisträgerin / der Preisträger wird an die Jahresversammlung der GGSP vom 30. August 2018 eingeladen, wo sie/er die Arbeit in einem Vortrag vorstellt und den Preis entgegennimmt.

Wir freuen uns auf Ihre Bewerbung!

Prof. Dr. med. Dr. phil. Paul Hoff, Präsident GGSP
Schweizerische Gesellschaft für Neuroradiologie SGNR

\section{Peter Huber Preis 2018}

Die Schweizerische Gesellschaft für Neuroradiologie schreibt zum Gedächtnis an Herrn Professor Dr. med. Peter Huber und zur Förderung des Fachgebietes der Neuroradiologie den Peter Huber Preis für eine experimentelle und/oder klinische Arbeit aus dem Gesamtgebiet der Neuroradiologie (allgemeine diagnostische Neuroradiologie, funktionelle Neuroradiologie, interventionelle Neuroradiologie, pädiatrische Neuroradiologie, Neuroradiologie der Kopf- und Halsregion) aus.

Der Erstautor sollte in neuroradiologischer Weiterbildung stehen oder eine neuroradiologische Weiterbildung abgeschlossen haben und nicht über 40 Jahre alt sein. Die eingereichte Arbeit darf 50 Seiten nicht überschreiten und muss in englischer oder deutscher Sprache verfasst sein. Die Arbeit sollte noch nicht ausgezeichnet worden sein und darf nicht an anderer Stelle zur Prämierung eingereicht sein. Pro Person kann nur eine Arbeit eingereicht werden.

Der gestiftete Preis ist mit 2000 CHF dotiert und wird im Rahmen der Jahrestagung vom 24. und 25. Mai 2018 in Lugano verliehen. Der Preisträger/die Preisträgerin wird zwei Wochen vor dem Kongress über die Entscheidung des Preiskomitees benachrichtigt und soll die Ergebnisse der Arbeit in einer 4-minütigen Zusammenfassung vorstellen. Der Preis ist teilbar.

Die Arbeiten sind per Mail (mit Abbildungen) bis zum 30. März 2018 an die Geschäftsstelle der Schweizerischen Gesellschaft für Neuroradiologie SGNR, c/o IMK Institut für Medizin und Kommunikation AG, einzureichen (sgnr[at]imk.ch).

Für den Vorstand der SGNR Prof. Dr. med. Luca Remonda, Präsident

\section{Sujet d'actualité en ligne -} www.bullmed.ch/fr/tour-dhorizon
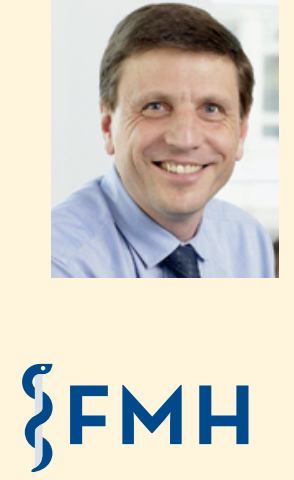

Interview de Franz Immer, directeur de Swisstransplant

\section{«Un registre est source de clarté et de sécurité»}

Pourquoi il est désormais temps, en Suisse aussi, de réfléchir à l'introduction du consentement présumé au sujet des dons d'organes.

\section{Le corps médical est ouvert à de nouveaux modèles de finance}

Etude représentative sur mandat de la FMH. 